\title{
In-situ Observation of Graphene Sublimation and Edge Reconstructions
}

\author{
Jian Yu Huang*, Liang Qi**, Ju Li**, Feng Ding***, Boris I. Yakobson****, Ping Lu***** \\ * $\quad$ Center for Integrated Nanotechnologies, Sandia National Laboratories, Albuquerque 87185 \\ ** Department of MSE, University of Pennsylvania, Philadelphia, Pennsylvania 19104, USA \\ *** Institute of Textiles and Clothing, Hong Kong Polytechnic University, Hong Kong, China \\ **** Department of ME \& MS, Rice University, Houston, Texas 77005 \\ ***** Sandia National Laboratories, Mail Stop 1411, Albuquerque, New Mexico 87185
}

We induced sublimation of suspended few-layer graphene by Joule-heating in-situ a transmission electron microscope (TEM) [1]. The sublimation front consisted of mostly $\{1100\}$ zigzag edges, and under appropriate conditions exhibited fractal-like "coastline" morphologies. Extensive multiplelayer reconstructions led to the formation of unique carbon nanostructures, such as $s p^{2}$-bonded bilayer edges (BLEs), $s p^{3}$ bilayer junctions, nanotubes connected to BLEs and tunneling multiple layers of graphene sheets, and flat fullerenes/nanopods. We reproduced the "coastline" sublimation morphologies by kinetic Monte Carlo simulations, revealing the unique geometrical and topological features of 2-dimensional (2D) graphene sublimation and reconstructions, enabled by bending, that are different from any first-order phase transformations of 3D bulk materials. These results indicate that multiple-layer graphene can offer unique opportunities for tailoring carbon-based nanostructures and engineering novel nano-devices with complex topologies.

Figure 1 shows a fractal-like sublimation pattern. Initially, a main crack (Fig. 1a) with some small secondary cracks appeared in one of the 5 double-layers. The main crack and its secondary cracks all grew with the lapse of time (Fig. 1b). Simultaneously, new cracks were nucleated on the side faces of existing ones and the propagation directions can change to new sets of zigzag planes. Similarly, the next generations of cracks nucleated and grew, until a fractal-like pattern emerged (Fig. 1c). The cracks propagated along one of the 6 equivalent $\{1100\}$ zigzag edges, and very rarely, a crack propagated along a $\{1120\}$ armchair edge. From the overlaid sequential images of the sublimation fronts (Fig. 1d), we calculated the fractal dimension of the "coastline" pattern to be 1.52 (Fig. 1e).

More than $90 \%$ of the graphene perimeters shown in Fig. 1 are in fact BLEs, with each lattice fringe in the high resolution TEM (HRTEM) images representing an edge of a double-layer graphene $[1,2]$. Figure 2 shows direct evidence of a bilayer structure. A void initially appeared on the top layer (Fig. 2a, 2e), and migrated towards the edge of the double-layer (Fig. 2b, 2f), then the bottom layer (shown in blue in Fig. 2) sublimated. As the bottom layer sublimated, its trailing edge bonded with the top layer (pointed out by green arrows in Fig. 2c, 2d, 2g), forming BLEs in-situ. The results indicate that a double-layer graphene rather than a single-layer graphene is the more stable structural unit in a multiple-layer graphene. The results have important implications in the device application of graphene: when one designs a graphene-based device, one must consider the possibility that the device operates with a BLE or $s p^{3}$ junction, rather than with a single-layer edge, even in the case of a monolayer graphene, as its edge is likely to curl up, forming a BLE plus a $s p^{3}$ junction.

\section{References}

[1] J.Y. Huang et al., (Submitted).

[2] Z. Liu et al., Physical Review Letters 102, (2009) 015501.

Acknowledgement: Sandia National Laboratories is a multi-program laboratory operated by Sandia Corporation, a Lockheed-Martin Company, for the U. S. Department of Energy under Contract No. DE-AC04-94AL85000. 

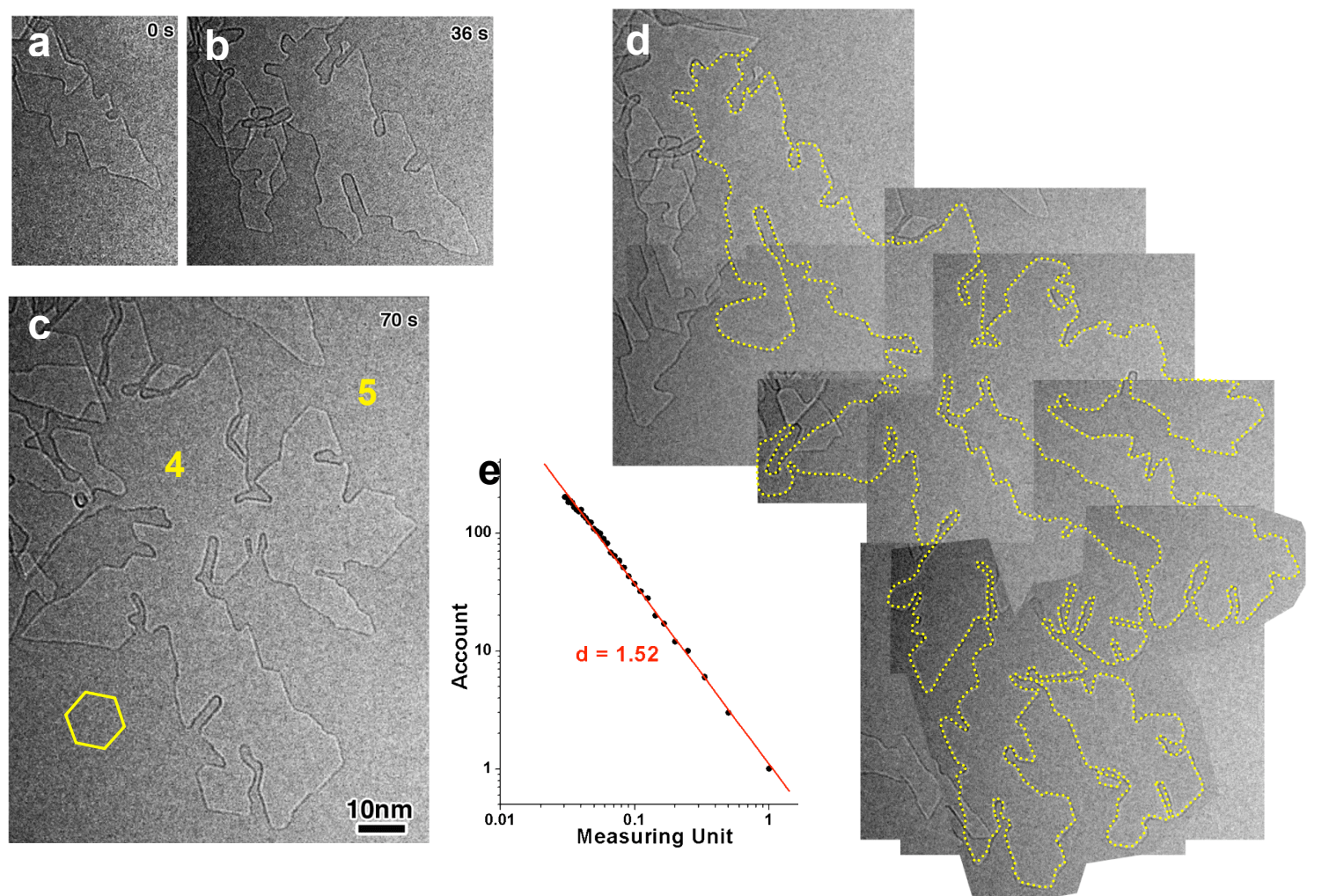

Fig. 1 (a-c) Sequential HRTEM images showing a fractal sublimation pattern of graphene. The numbers " 4 " and " 5 " indicate the layer thickness is 4 and 5 double-layers, respectively. The hexagon in Fig. 1c marks the 6 sets of equivalent $\{1100\}$ zigzag planes. (d) An overlay of sequential images of the propagating crack fronts with the crack edges highlighted in yellow-dotted lines. (e) A fractal dimension estimation of the yellow-dotted fractal pattern.
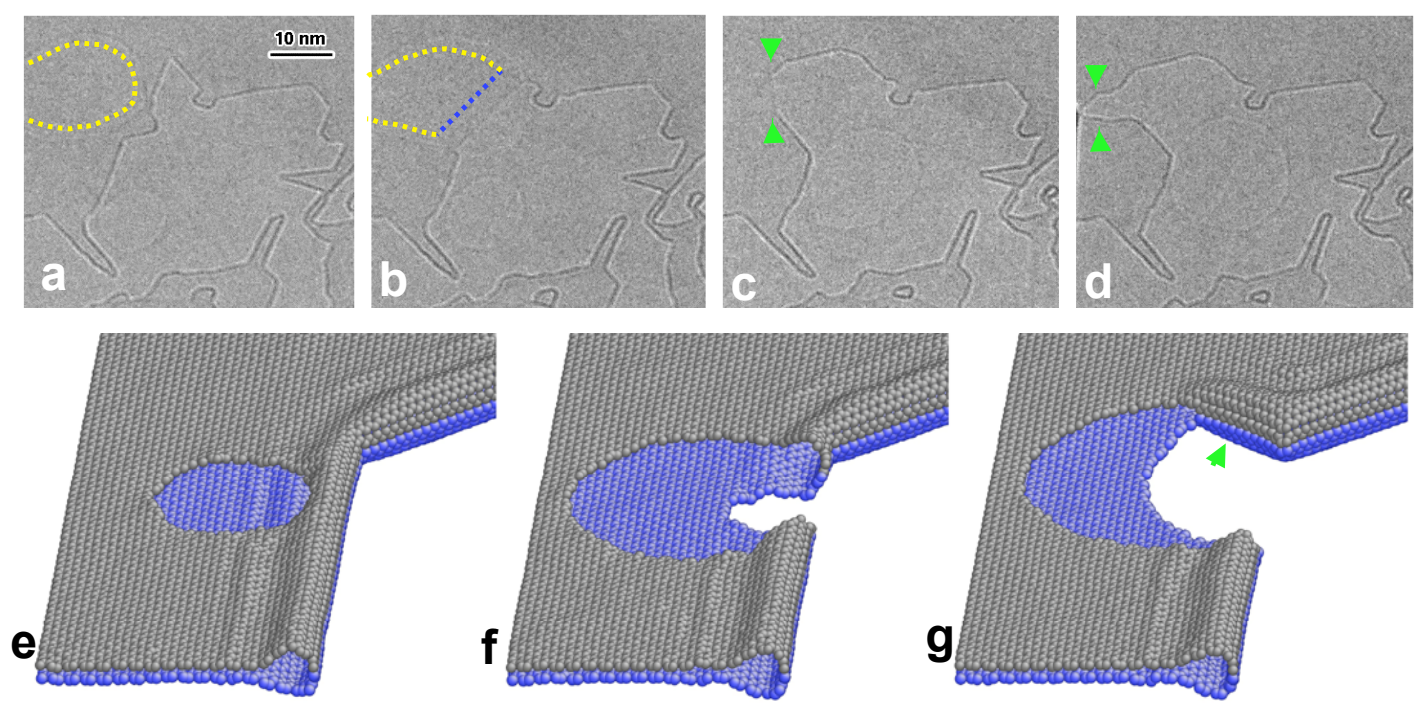

Fig. 2 (a-d) Sequential HRTEM images showing double-layer graphene. The yellow hexagon marks the six sets of zigzag planes. Yellow-dotted lines point out the edge of a vacancy hole on the top-layer. The blue-dotted line pointed out the edge of a single-layer graphene. Green arrows denote the reconstructed double-layer graphene. (e-f) Schematic drawings corresponding to (a-c), respectively. 